\title{
INFLUÊNCIA DOS FATORES DE RISCO NA PATOGÊNESE DA HIPERTENSÃO ARTERIAL SISTÊMICA
}

Gustavo Pires Monteiro, Marco Tulio Marins Franco, Matheus Claudio Magalhães Silva, Moacir Ferreira Junior. Influência dos fatores de risco na patogênese da hipertensão arterial sistêmica. Revista Saúde Dinâmica, vol. 3, núm.1, 2020. Faculdade Dinâmica do Vale do Piranga. 


\title{
Influência dos fatores de risco na patogênese da hipertensão arterial sistêmica
}

\section{Influence of the risk factors in the pathogenesis of the systemic arterial hypertension}

\author{
Gustavo Pires Monteiro ${ }^{1}$, Marco Tulio Marins Franco $^{I}$, Matheus Claudio Magalhães Silva ${ }^{\text {, }}$ \\ Moacir Ferreira Junior ${ }^{1}$ \\ ${ }^{I}$ Curso de Medicina, Faculdade Dinâmica do Vale do Piranga, Rua G, 205, Bairro Paraíso, Ponte \\ Nova-MG, Cep: 35430-302, gustavopmonteiro11@gmail.com
}

\section{Resumo}

A hipertensão arterial caracteriza-se pela elevação da pressão sanguínea, configurando-se como um dos principais problemas de saúde e constitui-se como um dos maiores fatores de risco para doenças cardiovasculares. O objetivo deste estudo é descrever e discutir sobre a influência dos fatores de risco na patogênese da hipertensão arterial sistêmica, tal como enfatizar a importância desse conhecimento visto a necessidade de abordagem correta, sendo ela primeiramente comportamental diminuindo assim os fatores de risco conhecidos, e, se necessário a utilização farmacológica diminuindo assim a prevalência e as complicações dessa doença. Dentre os fatores de risco, eles destacam-se: Obesidade, sedentarismo, idade, raça, estresse, etilismo, tabagismo, Diabetes Mellitus e dislipidemia. Elaborado a partir de uma revisão bibliográfica com as seguintes bases de dados: Pubmed, Scielo, Bireme, Sociedade Brasileira de Cardiologia e Sociedade Portuguesa de Cardiologia.

Palavras-chave: Fatores de risco; patogênese; hipertensão arterial.

\begin{abstract}
The systemic arterial hypertension is characterized by the increase of blood pressure, it sets up as one of the main health problems and it is also considered a big risk factor for cardiovascular diseases. The objective of this study is to describe the influence of the risk factors in the pathogenesis of the systemic arterial hypertension, such as to emphasize the importance of this knowledge so that the risk factors are decreased, and, if necessary, the pharmacological usage so that the prevalence and incidence of the systemic arterial hypertension also decreases. Obesity, dyslipidemia, age, race, alcoholism, stress, smoking, Diabetes Mellitus and sedentarism are some examples of those risks. It was drafted by a bibliographic review with the following databases: Pubmed, Scielo, Bireme, Sociedade Brasileira de Cardiologia e Sociedade Portuguesa de Cardiologia.
\end{abstract}

Keywords: Risk factors; pathogenesis; systemic arterial hypertension. 


\section{INTRODUÇÃO}

Atualmente as doenças cardiovasculares têm sido a maior causa de mortes em todo o mundo, sendo responsáveis por mais de 17 milhões de óbitos em 2008. A Organização Mundial da Saúde estima que, em 2030, quase 23,6 milhões de pessoas morrerão decorrentes destas doenças (RADOVANOVIC et. al., 2014). Dentre elas, a hipertensão arterial sistêmica, (HAS) a qual é definida como "pressão sanguínea arterial elevada" (CECIL, 2009), é considerada um problema de saúde pública mundial e constitui um dos mais importantes fatores de risco para complicações cardíacas e cardiovasculares (MACHADO, 2012; MION, 2002). A maioria desses acometimentos ocorre em indivíduos com leves alterações nos fatores de risco que, se deixados sem tratamento, podem desencadear condições patológicas mais graves (MARIN, 2016). A prevenção e o controle da HAS apresentam uma grande importância para todas as nações, por ser uma doença crônica, que necessita de acompanhamento e tratamento por toda a vida (NOBRE, 2010).

No Brasil, a prevalência da HAS é de aproximadamente $30 \%$ da população total (TEIXEIRA, GOULART, BUSNELLO, PELLANDA, 2016). A sétima Diretriz Brasileira de Hipertensão Arterial estabelece a hipertensão arterial (HA) como uma condição clínica multifatorial caracterizada pela elevação sustentada dos níveis pressóricos $\geq 140$ e/ou 90 $\mathrm{mmHg}$. Ela frequentemente associa-se a distúrbios metabólicos, alterações funcionais e/ou estruturais de órgãos-alvo, sendo agravada pela presença de outros fatores de risco (FR) como tabagismo, ingestão excessiva de álcool, sedentarismo, estresse, idade, Diabetes Mellitus, dislipidemia, sexo (maior acometimento em homens), gênero, raça (negros) estão intimamente relacionados com a HAS (SIMONETTI, BATISTA, CARVALHO, 2002). Uma dieta desbalanceada e rica em sódio está também associada à doença, sendo um dos principais fatores de risco modificáveis para o controle da doença, aliado à prática regular de atividades físicas, assim como cessar o tabagismo e o alcoolismo. (TEIXEIRA, 2016). Além disso, a HAS não controlada é um importante fator de risco para o desenvolvimento de doenças cardiovasculares, como as cardiopatias isquêmicas (infarto agudo do miocárdio) e, lesões no sistema nervoso central (acidente vascular cerebral, aterosclerose e trombose) (NOBRE, 2010).

Dessa maneira, como foi exposto, existem vários trabalhos que relacionam inúmeros fatores de risco na incidência da HAS; entretanto, existem poucos que demonstram o conhecimento da etiopatogênese desses fatores no aparecimento de tal enfermidade. Assim 
sendo, o objetivo deste trabalho é explorar e esclarecer sobre a relação dos fatores de risco na gênese da HAS.

\section{MATERIAIS E MÉTODOS}

Esse estudo foi feito a partir de uma revisão bibliográfica de trabalhos científicos que buscaram esclarecer os fatores de riscos para a HAS e o que eles implicam na gênese desta moléstia.

A revisão bibliográfica foi realizada a partir das bases de dados: o Pubmed, Scientific Electronic Library Online (SciELO), Centro Latino-Americano e do Caribe de Informação em Ciências da Saúde (Bireme), diretriz da Sociedade Brasileira de Cardiologia e Sociedade Portuguesa de Cardiologia. Os artigos escolhidos estavam publicados em inglês e português, com limite de 30 anos, pelo fato de a hipertensão arterial sistêmica ser um assunto que está em constante atualização. Os principais descritores utilizados para busca da revisão bibliográfica foram: Fatores de risco, patogênese e hipertensão arterial.

Foram selecionados 28 artigos para o estudo em questão, sendo que a coleta dos artigos ocorreu em um período de 02 meses de buscas, tendo como critérios de exclusão o tempo de publicação dos artigos, e critérios de inclusão artigos recentes que mostrassem a forma, causas e consequências da hipertensão arterial.

\section{RESULTADOS E DISCUSSÃO}

O mecanismo pelo qual o álcool se relaciona com o aumento da pressão arterial está no fato dele ser capaz de ativar o sistema nervoso central, o sistema nervoso autônomo (simpático) e o sistema renina-angiotensina-aldosterona, por meio da indução do cortisol plasmático (ZALESKIL, 2004). Quantidades baixas de ingestão diária de álcool (1 a 2 doses) são insuficientes para elevá-la (CECIL, 2009).

O tabagismo, por sua vez, causa um aumento agudo da pressão arterial e da frequência cardíaca, mediado pela nicotina que age como um agonista adrenérgico, promovendo a liberação local e sistêmica de catecolaminas (vasopressina, dopamina, norepinefrina) (PINTO, 2010). 
Considera-se também a idade como um fator de risco importante para a HAS, uma vez que a musculatura lisa e o tecido conjuntivo dos vasos são alterados como consequência do envelhecimento. (PESSUTO, 1998).

Características psicológicas como o estresse crônico podem, de fato, elevar a pressão arterial por meio da ativação do sistema nervoso simpático e das vias neuroendócrinas. $\mathrm{O}$ sistema nervoso simpático (SNS) controla a maioria das respostas do organismo a agentes estressores. Desse modo, além da produção de epinefrina, norepinefrina e acetilcolina, o SNS secreta diversos neuropeptídeos como somatostatina, galanina, neurotensina, encefalina, que são influenciados pelo Sistema Nervoso Central e interagem com o eixo hipotálamohipofisário, regulando a resposta ao estresse. Por fim, os glicocorticóides têm papel regulatório na atividade basal desse eixo e nas respostas finais ao estresse pela existência de um mecanismo de feedback negativo, regulando todo o processo (VIEIRA, 2007).

A obesidade, sobretudo a abdominal, está associada a um perfil lipídico aterogênico com aumento no colesterol-lipoproteína de baixa densidade (LDL) e nos níveis da adipocina vascular protetora adiponectina (CARNEIRO, 2003). O aumento do LDL é responsável pela formação de placas ateromatosas que obstruem a luz do vaso e aumentam a resistência vascular (MARTINS, 1997). A hipertensão induzida pela obesidade pode causar elevação da resistência vascular periférica e do débito cardíaco, aumento no tônus do sistema nervoso simpático, exacerbação da sensibilidade ao sal e retenção de sal; com frequência, a hipertensão melhora após uma pequena perda ponderal (TEIXEIRA, 2016).

Já o Diabetes Mellitus tipo II, que cursa com hiperinsulinemia, ocorre uma grande retenção de água e sódio nos túbulos renais, o que aumenta a atividade da bomba $\mathrm{Na}+\mathrm{H}+$ na musculatura lisa dos vasos e leva ao incremento do cálcio e do $\mathrm{pH}$ intracelular, aumentando a proliferação celular (CAROLINO, 2008). Além disso, ocorre um grande estímulo à atividade simpática, aumentando os níveis arteriais pressóricos, por meio, principalmente, das catecolaminas. O Diabetes Melito tipo 2, principalmente, cursa com defeito metabólico relevante à patogênese da hipertensão arterial, tal como a resistência à insulina (FURTADO 2007). Esta, por sua vez, resulta em acúmulo de ácidos graxos livres no fígado e nos músculos estriados, ocorrendo o aumento da oxidação destes ácidos graxos que danificam o DNA nuclear das células endoteliais arteriais, gerando dano macrovascular ao endotélio e gerando aumento dos níveis pressóricos por meio da liberação de fatores pró-inflamatórios (MARTINS, 1997). 
O uso da variável raça como atributo essencial para a definição da hipertensão arterial sistêmica toma como base uma hipótese de suscetibilidade apoiada em dois pressupostos - a existência de um alelo ou grupo de alelos que levariam a uma suscetibilidade aumentada à hipertensão e uma maior prevalência desses alelos entre as pessoas negras quando comparadas com indivíduos brancos (KLAG et al., 1991). Por outro lado, a associação entre pigmentação da pele e hipertensão sugeriria que a melanina estaria envolvida no mecanismo bioquímico que eleva a hipertensão arterial sistêmica e que, quanto mais escura a pele, maior a presença desses alelos da suscetibilidade (MURRAY, 1991).

O sedentarismo, por sua vez, constitui-se como um dos principais fatores de risco para a hipertensão arterial sistêmica. Este fato corrobora-se, pois, a atividade física provoca importantes adaptações hemodinâmicas e autonômicas que vão influenciar o sistema cardiovascular, objetivando manter a homeostasia celular diante do incremento das demandas metabólicas. Além disso, há elevação do débito cardíaco, redistribuindo o fluxo sanguíneo e aumentando a perfusão para os músculos em atividade. Ademais, a atividade física expande o volume plasmático, melhora a função endotelial e potencializa a sensibilidade insulínica na musculatura esquelética (RONDON, 2003).

\section{CONCLUSÃO}

De acordo com a revisão dos artigos, concluímos que o tabagismo, ingestão excessiva de álcool, sedentarismo, estresse, idade, Diabetes Mellitus, dislipidemia, sexo, gênero, raça são fatores de risco que influenciam na patogênese da HAS, levando a um pior prognóstico da doença. Sendo assim, esse conhecimento é crucial para o seu diagnóstico e manejo, contribuindo para um melhor entendimento desta condição no intuito de melhorar a qualidade de vida da população afetada. É importante ressaltar que fatores de riscos como tabagismo, estresse, sedentarismo e obesidade são modificáveis com mudanças nos hábitos de vida; sendo assim, constituem a primeira linha de tratamento não farmacológico para a HAS, podendo associar a monoterapia em casos refratários (MALACHIAS, 2016). No entanto, o estudo limitase pela escassez de referências bibliográficas recentes que descrevam com consistência a patogênese da hipertensão arterial sistêmica. 


\section{REFERÊNCIAS}

AGUIAR, A.P.S.; OLIVEIRA, M.R.; BARRETO, N.A.M.P.; FERREIRA, S.F.V.; FERREIRA, C.M.M.; PINHO, L. Hipertensão e dislipidemias em pacientes Diabetes Mellitus tipo2: uma revisão integrativa. Renome: Revista Norte Mineira de Enfermagem. v. 4, n. 1, 2015.

BRASIL. Ministério da Saúde. Sistema de cadastramento e acompanhamento de hipertensos e diabéticos, 2002. Disponível em <http://hiperdia.datasus.gov.br/>. Acesso em: 16 de Maio de 2018.

CIPULLO, J.P.; MARTIN, J.F.V.; CIORLIA, L.A. de S.; GODOY, M.R.P. de; CAÇÃO, J.C.; LOUREIRO, A.A.C.; BURDMANN, E.D.A. Prevalência e Fatores de Risco para Hipertensão em uma População Urbana Brasileira. Arquivos Brasileiros de Cardiologia, 94(4), 519-26, 2010.

CARNEIRO, G. et al. Influência da distribuição da gordura corporal sobre a prevalência de hipertensão arterial e outros fatores de risco cardiovascular em indivíduos obesos. Revista da Associação Medica Brasileira. São Paulo, v. 49, n. 3, p. 306-311, jul./set., 2003.

CAROLINO, I.D.R. et al. Fatores de risco em pacientes com diabetes mellitus tipo 2. Revista Latino-Americana de Enfermagem (online), Ribeirão Preto, v. 16, n. 2, p. 238-244, mar./abr. 2008.

COSTA, J.S.D.; BARCELlOS, F.C.; SCLOWITZ, M.L.; SCLOWITZ, I.K.T.; CASTANHEIRA, M.; OLINTO, M.T., et al. Prevalência de hipertensão arterial em adultos e fatores associados: um estudo de base populacional urbana em Pelotas, Rio Grande do Sul, Brasil. Arquivos Brasileiros de Cardiologia. Versão online Vol.88 no.1 São Paulo Jan. 2007. < https://doi.org/10.1590/S0066-782X2007000100010 >

FERRANNINI, E.; CUSHMAN, W.C. Diabetes and hypertension: the bad companions. The Lancet. Volume 380, Edição 9841, Páginas 601-610, 2012.

FREITAS, L.R.S.; GARCIA, L.P. Evolução da prevalência do diabetes e deste associado à hipertensão arterial no Brasil: análise da Pesquisa Nacional por Amostra de Domicílios, 1998, 2003 e 2008. Revista Epidemiologia e Serviços de Saúde, versão online v.21 n.1 Brasília mar. 2012. < http://dx.doi.org/10.5123/S1679-49742012000100002>

FURTADO, M.V.; POLANCZYK, C. A. Cardiovascular prevention in diabetic patients: an evidenced-based review. Arquivos Brasileiros de Endocrinologia \& Metabologia. São Paulo, v. 51, n. 2, p. 312-318, mar. 2007.

GOLDMAN, L., AUSIELlO, D. Cecil: Tradado de Medicina interna. 23 ${ }^{\mathrm{a}}$ edição. Rio de Janeiro, RJ: Elsevier Saunders, 2009.

HARTMANN, M.; COSTA, J.S.D.; OLINTO, M.T.A.; PATTUSSI, M.P.; TRAMONTINI, A. Prevalência de hipertensão arterial sistêmica e fatores associados: um estudo de base 
populacional em mulheres no Sul do Brasil. Cadernos de Saúde Pública, vol.23 no.8 Rio de Janeiro 23(8), 1857-1866, 2007.

HDS - Hypertension in Diabetes Study Group. HDS 1: Prevalence of hypertension in newly presenting type 2 diabetic patients and association with risk factors for cardiovascular and diabetic complications. Journal of Hypertension, London, v.11, n.3, p.309-17, 1993.

KLAHG, M.J. et al. The association of skin color with blood pressure in US Blacks with low socioeconomic status. Journal of American Medical Association, v. 265, n. 5, p. 599-602, 1991.

MACHADO, M.C.; PIRES, C.G.S. \& LOBÃO, W.M. Concepções dos hipertensos sobre os fatores de risco para a doença. Ciência \& Saúde Coletiva, 17(5), 1365-1374, 2012

MARIN, N.S.; SANTOS, M.F.; MORO, A.D.S. Perception of hypertensive patients about their non-adherence to the use of medication. Revista Da Escola de Enfermagem Da USP, 50 Spec, 61-7, 2016.

MALACHIAS, M.V.B et al. $7^{\text {a }}$ Diretriz Brasileira de Hipertensão Arterial. Arquivos Brasileiros de Cardiologia, Rio de Janeiro, v. 107, n. 3, supl. 3, p. 1-104, set. 2016.

MARTINS, I.S. et al. Atherosclerotic cardiovascular disease, lipemic disorders, hypertension, obesity and diabetes mellitus in population of a metropolitan area of southeastern Brazil: III _ Hypertension. Revista de Saúde Pública, São Paulo; v.31, n.5, p.466-71, 1997.

MENGESHA, A.Y. et al. Hypertension and related risk factors in type 2 diabetes mellitus (DM) patients in Gaborone City Council (GCC) clinics, Gaborone, Botswana. African Health Sciences, v. 7, n. 4, p. 244-5, 2007.

MION JR, D. et al. IV Diretrizes Brasileiras de Hipertensão Arterial, 2002b. Disponível em: http://www.sbn.org.br/diretrizes. SBD (Sociedade Brasileira de Diabetes) - Consenso Brasileiro sobre Diabete 2002. Diagnóstico e classificação do diabetes melito e tratamento do diabetes melito do tipo 2 . Disponível em $<$ http://www.diabetes.org.br/educacao/docs/consenso_atual_2002>. Acesso em 20 de Maio de 2018.

MURRAY, R.F. Skin color and blood pressure. Genetics or environment? Journal of American Medical Association, v. 265, n. 5, p. 639-40, 1991.

NILSSON, P.M.; CEDERHOLM, J. Diabetes, hypertension, and outcome studies: overview 2010. Diabetes Care; 34 (suplemento 2): S109 - S113, maio 2011.

NOBRE, F.; RIBEIRO, A.B.; MION, D. Control of arterial pressure in patients undergoing anti-hypertensive treatment in Brazil: Controlar Brazil. Arquivos Brasileiros de Cardiologia, 94(5), 663-670, 2010. 
PASSOS, V.Ma. D.A.; ASSIS, T.D.; BARRETO, S.M. Hipertensão arterial no Brasil: estimativa de prevalência a partir de estudos de base populacional. Epidemiologia E Serviços de Saúde, 15(1), 35-45, 2006.

PESSUTO, J.; CARVALHO, E.C. Fatores de risco em indivíduos com hipertensão arterial. Revista Latino-Americana de Enfermagem, 6(1), 33-39, 1998.

PINTO M.; UGÁ MAD. Os custos de doenças tabaco-relacionadas para o Sistema Único de Saúde. Cadernos de Saúde Pública. Página 1234, 2010.

RADOVANOVIC, C.A.T.; SANTOS, L.A.Dos; CARVALHO, M.D.de B.; MARCON, S.S. Arterial Hypertension and other risk factors associated with cardiovascular diseases among adults. Revista Latino-Americana de Enfermagem, 22(4), 547-553, 2014.

RONDON, M.U.P.B.; BRUM, P.C. Exercício físico como tratamento não farmacológico da hipertensão arterial. Revista Brasileira de Hipertensão, 10: 134-139, 2003.

SIMONETTI, J.P.; BATISTA, L.; CARVALHO, L.R.de. Hábitos de saúde e fatores de risco em pacientes hipertensos. Revista Latino-Americana de Enfermagem, 10(3), 415-422, 2002.

TEIXEIRA, J.D.F.; GOULART, M.R.; BUSNELLO, F.M.; \&PELLANDA, L.C. Hypertensives' Knowledge about High-Sodium Foods and Their Behavior. Arquivo Brasileiro de Cardiololiga, vol.106 no.5 São Paulo May 2016.

VIEIRA, F.L.H.; LIMA, E.G. Testes de estresse laboratoriais e hipertensão arterial. Revista Brasileira de Hipertensão, vol.14(2): 98-103, 2007.

ZALESKIL, M.; MORATO, G.S.; SILVA, V.A.; LEMOS, T. Aspectos neurofarmacológicos do uso crônico e da Síndrome de Abstinência do Álcool. Revista Brasileira de Psiquiatria, vol.26 suppl.1 São Paulo May, 2004.

\section{Declaração de Interesse}

Os autores declaram não haver nenhum

conflito de interesse

\section{Financiamento}

Financiamento próprio.

\section{Agradecimentos}

Agradecemos a todos, que direto ou indiretamente fizeram parte para a elaboração desse artigo, em especial ao nosso Orientador Moacir Ferreira Junior pelo incentivo e paciência na elaboração do nosso trabalho.

\section{Colaboração entre autores}

Ferreira Junior assessorou a elaboração e execução do projeto, Monteiro, Franco e Silva atuaram na elaboração do projeto, coleta de dados e redação do texto. Os autores citados participaram da concepção, bem como da revisão que resultou neste artigo. Os autores assumem a responsabilidade pelo conteúdo e pela originalidade do manuscrito e afirmam que os resultados do manuscrito não são plagiados. 